\title{
LA IMAGEN DE AMÉRICA LATINA EN LA REVISTA CUADERNOS HISPANOAMERICANOS (1948-1951)
}

\author{
ANNA Virágh
}

Universidad de Szeged

\section{The image of Latin America in the magazine Cuadernos Hispano- americanos (1948-1951)}

The paper presents the way the independence of the Latin American countries, their relations with Spain and their future perspectives were represented in the first volumes of Cuadernos Hispanoamericanos, a cultural magazine of propagandistic aims established by the Franioist government in 1948. In the aftermath of the Seiond World War, the Francoist regime, forced into a relatively extended international isolation by the resolutions of the UN, had to tone down its international propaganda and seek allies for its cause, resulting in a rapprochement towards Latin American countries. Cuadernos Hispanoamericanos was a more sophisticated means of this propaganda, although it also had the important merit of encouraging a real dialogue between Latin American and Spanish intellectuals and artists. The authors of the magazine retained the principal characteristics of the official ideology of Hispanidad, but also argued for a more balanced relationship between Latin America and Spain, and saw Latin America as an emerging power witbin the international sphere.

América Latina permaneció un elemento central de la identidad histórica y cultural de España también después de la independencia de los paises del subcontinente. A lo largo del siglo XX, las interpretaciones acerca de las relaciones entre América y España variaron en las diversas etapas de la evolución política del país peninsular, y diferentes matices cobraron relevancia o fueron confinados al trasfondo del proceso político e ideológico de la península. En la primera fase de la dictadura franquista, hasta mediados de la década de los cuarenta, el régimen adoptó el concepto de la Hispanidad, desarrollado por el ideólogo Ramiro de Maeztu, como fundamento de su diplomacia cultural y de sus reivindicaciones de poder y protagonismo en la región latinoamericana. Según la ideología de la Hispanidad, la tradición cultural y espiritual compartida por los países de América Latina y su antigua metrópoli consolidó un fundamento sólido para la creación de una comunidad permanente de todos los pueblos hispanos, con la vocación de vigilar sobre los valores del catolicismo, luchar contra el comunismo y estar a la vanguardia de la evolución histórica del Occidente como ejemplo del poder salvador de la verdadera $\mathrm{fe}^{1}$.

${ }^{1}$ Ramiro MAEZTU, Defensa de la Hispanidad, Valladolid, 1938, 35-37. 
En este contexto ideológico, la imagen de América Latina y de sus relaciones con España fue muy esquemática, de 'blanco y negro': en la prensa y la propaganda española de la época, el subcontinente apareció como una región mutilada por la Independencia, un recordatorio vivo de la pérdida de la gran obra de España del glorioso pasado imperial2. Asimismo, las interpretaciones acerca del futuro de los países de América Latina estaban centradas en los esfuerzos contemporáneos de España para la creación de una nueva construcción de confeceración hispanoamericana, bajo el liderazgo del país peninsular ${ }^{3}$. Sin embargo, con el fin de la Segunda Guerra Mundial, el gobierno franquista, cada vez más aislado en el escenario internacional por su origen poco legítimo y por sus explícitas inclinaciones pro-Eje, fue obligado a a suavizar el tono de su propaganda y reconsiderar las líneas de acción de su política exterior en general, y hacía América Latina en particular. Esta moderada apertura ideológica también se manifestó en la creación de varias nuevas instituciones y organismos culturales, destinadas a renovar la propaganda exterior y la diplomacia cultural del régimen; entre ellos, de la revista Cuadernos Hispanoamericanos, fundada en 1948.

El presente ensayo examina de qué manera los temas relacionados con la Independencia latinoamericana, los lazos entre el subcontinente y España y con la imagen del futuro de América Latina fueron representados y evaluados en los primeros volúmenes de la revista, publicados en el proceso de la consolidación de la apertura ideológica del régimen franquista. Se puede afirmar sin exageración que esta publicación, también existente en la actualidad, aportó un espacio cultural moderado en contraste con las anteriores publicaciones de carácter propagandístico. Cuadernos Hispanoamericanos era la primera revista de la época que ofrecía un foro internacional, aunque de límites ideológicos relativamente estrechos, para renombrados ideólogos, intelectuales y artistas de España y de América Latina para determinar las particularidades esenciales de la identidad cultural y política de América Latina y de España, e identificar su situación en el nuevo orden cultural e ideológico después de la Segunda Guerra Mundial.

Para el periodo de la publicación del primer número de Cuadernos Hispanoamericanos, en los primeros meses de 1948, simultáneamente a la agravación de la Guerra Fría, la situación internacional de España había mejorado considerablemente. Aunque seguía vigente la cuarentena diplomática relativamente extendida, impuesta al régimen por la resolución del diciembre de 1946 de la Asamblea General de Naciones Unidas, la mayoría de las democracias de Occidente desarrollaron una política práctica más flexible con el régimen franquista ${ }^{4}$. Su discurso público internacional mantuvo las enérgicas condenas públicas contra las numerosas características antidemocráticas del régimen, pero en línea con las consideraciones pragmatistas impuestas por la Guerra

2 Monsterrat HUGUET SANTOS, "América Latina en la prensa del primer franquismo", in: Montserrat HUGUET, A. NIÑO et al. (eds.), La formación de la imagen de América Latina en España, 1898-1989, Madrid, 1992, 349-350.

${ }^{3}$ Ibidem, 353.

4 Julio GIL PECHARROMÁN, La politica exterior del franquismo. Entre Hendaya y El Aaiun, Barcelona, Flor del viento, 2008, 173-174. 
Fría, no dudaron en asegurar al Caudillo de que apoyar la estabilidad a largo plazo de su régimen coincidía con sus intereses estratégicos. Como resultado de las circunstancias externas, en la esfera de la política exterior, España adoptó la postura de la llamada política de la dignidad (concepto también fomentado por la propaganda del régimen) ${ }^{5}$, preparándose para una posible larga espera hasta que España llegara a ser un miembro plenamente aceptado e integrado de la sociedad internacional. Como consecuencia, hasta que el país fue admitido en el seno de la Organización de las Naciones Unidas en 1955 , la meta principal de la política exterior española era encontrar aliados para su causa. Fue en este período que las relaciones basadas en el pasado común y la herencia cultural compartida fueron revalorizadas y se han convertido más intensivas con los países del Oriente Medio, y especialmente, con América Latina ${ }^{6}$.

Como resultado de este proceso de acercamiento, la diplomacia cultural y la propaganda del régimen franquista eran especialmente intensivas en relación con los países latinoamericanos. Como ya hemos mencionado, el hilo conductor ideológico de esta orientación era el concepto de la Hispanidad, y sus componentes: la preservación y la eventual restauración del grandioso pasado imperial y la vocación de mantener las tradiciones culturales y espirituales compartidas con los paises latinoamericanos. En 1940, el gobierno español fundó el Consejo de la Hispanidad, órgano político-cultural que perteneció al Ministerio de Asuntos Exteriores, para coordinar la política exterior del régimen hacía América Latina. El consejo directivo del organismo era formado por delegados del gobierno español, de la Falange y por figuras importantes de la vida científica española; además, la Iglesia católica también fue representada con sacerdotes de alto cargo ${ }^{7}$. La tarea principal del Consejo de la Hispanidad era intensificar las relaciones culturales con los países latinoamericanos y desarrollar una propaganda más eficaz para contrarrestar la opinión internacional negativa contra el régimen franquista. No obstante, en la práctica, el organismo especializado no llegó a ser efectivo. Su vocación de restablecer la comunidad de destino de los pueblos hispánicos despertó sospechas de un imperialismo encubierto incluso entre los políticos e intelectuales de la derecha latinoamericana, y la marcada presencia de la Falange en la organización tampoco ayudó a mantener el imagen de una institución predominantemente cultural ${ }^{8}$. Además, el tono paternalista de su política cultural tampoco resultó atractivo en América Latina?.

En 1945, el gobierno español reorganizó el Consejo de la Hispanidad en un nuevo órgano de política cultural y de propaganda, en el Instituto de Cultura Hispánica, adoptando un enfoque más moderado y métodos más modernos para impulsar las

\footnotetext{
` Lorenzo Delgado GÓMEZ-ESCALONILLA, Imperio de Papel. Acción cultural y política exterior durante el primer franquismo, Madrid, CSIC, 1992, 438.

${ }^{6}$ Raymond CARR, (coord.) Historia de España, tom. XLI. La época de Franco, Madrid, EspasaCalpe, 1996, 251-253.

${ }^{7}$ Jefatura del Estado, Ley de 2 de noviembre de 1940 por la cual se crea el Consejo de la Hispanidad, Madrid, Uguina, 1940, 5-8.

${ }^{8}$ GÓMEZ-ESCALONILLA, op. cit., 269-270.

9 Ibidem, 271.
} 
relaciones culturales entre España y los países de América Latina ${ }^{10}$. Aunque el mantenimiento de los vínculos espirituales entre todos los pueblos que componen la comunidad cultural de la Hispanidad permaneció la meta principal del organismo, su orientación práctica estaba basada en una relación más igual con los países latinoamericanos, dejando de lado el paternalismo ideológico del período anterior ${ }^{11}$. El Instituto creó un extensivo sistema de becas para fundar, sobre todo, la estancia de estudiantes universitarios latinoamericanos en universidades españoles, pero también ofreció becas para universidades de América Latina. Además, el Instituto contaba con un departamento autónomo propio en la Universidad de Madrid, con un colegio en la Universidad de Salamanca, y abrió varios centros en las capitales y las grandes ciudades de América Latina también. No obstante, es probable que la función del Instituto a través de la cual que ha llegado a los grupos más amplios de la sociedad española y latioamericana era su actividad editorial, con la publicación de numerosos autores afines a la ideología oficial del franquismo ${ }^{12}$. Aparte de estos tomos, la actividad editorial del Instituto también intensificó con la publicación de su propia revista cultural, Cuadernos Hispanoamericanos, a partir de 1948.

Aunque Cuadernos Hispanoamericanos oficialmente era una revista cultural, la matiz política y los objetivos propagandísticos eran evidentes en su selección de temas y en su funcionamiento. La revista representaba una poderosa herramienta de la propaganda cultural internacional de España después de la Segunda Guerra Mundial, en medio de un contexto de aislamiento internacional relativamente extendido, ya que su orientación cultural subrayaba la vocación latinoamericana del pais y sus importantes lazos culturales con los países de la región. En este aspecto, la revista ayudó a elevar la vocación de misión de la ideologia oficial franquista al nivel internacional.

Sin embargo, debido a su estructura y su filosofia editorial y prácticas de publicación, Cuadernos Hispanoamericanos era más que un simple portavoz de la propaganda franquista dirigida principalmente hacía el extranjero. Ya en su etapa principal, la revista contó con 63 autores y contribuidores permanentes y 13 ilustradores en España y en América Latina ${ }^{13}$. En su estructura, los temas y noticias culturales eran dominantes, pero también eran tecurrentes los de carácter político. La revista contaba con cinco secciones permanentes: "Del ser y pensar hispánicos", dedicada al tema de la identidad hispana; "Nuestro Tiempo", la cual abordaba temas de la actualidad, sobre todo, de política; "Arte y poética", sección de poesia; "Asteriscos", de noticias y reportajes breves sobre diferentes eventos y actividades culturales; y "Brújula para leer", con reseñas de obras, principalmente de carácter divulgativo ${ }^{14}$. Desde nuestro punto de vista, es importante

10 José Luis NELIA HERNÁNDEZ, "Hispanoamérica en el imaginario de ultramar de la política exterior franquista", in: Tzintzun, Revista de Estudios Históricos, 37, Universidad Michoacana de San Nicolás de Hidalgo, 2003, 82-83.

11 GÓMEZ-ESCALONILLA, op. cit., 451.

12 María Elena CAPELATO, "Cuadernos Hispanoamericanos: Idéias políticas numa revista de cultura", in: Varia Historia, vol. 21., 34, Belo Horizonte, 2005, 345.

${ }^{13}$ CAPELATO, op. cit., 346.

${ }^{14}$ Ibidem, 357. 
subrayar la introducción de otra sección permanente en 1951, titulada "¿Adónde va Hispanoamérica?", dedicada exclusivamente a las perspectivas futuras del mundo hispano ${ }^{15}$. La revista también editó números especiales temáticos de literatura con regularidad sobre la obra no sólo de Ramiro Maeztu, sino también autores más distantes de la ideología franquista como Jorge Luis Borges, Rainer Maria Rilke, Stéphane Mallarmé, o Ezra Pound ${ }^{16}$.

Hasta 1951, el director de Cuadernos Hispanoamericanos era un ideólogo renombrado del fascismo español, Pedro Laín Entralgo. Era natural que la revista, una herramienta importante de la política y diplomacia cultural del franquismo, contara con un liderazgo fiable. Sin embargo, hay que subrayar que Laín Entralgo perteneció a la corriente moderada, aperturista de la Falange. Anteriormente a la dirección de Cuadernos Hispanoamericanos, ya fue encargado a participar en la creación de una revista de propaganda de alta calidad, El Escorial, en la editorial de la cual, según las palabras del mismo Laín Entralgo, "la calidad primó sobre la voluntad de convencer"17. La extendida actividad científica de Lain Entralgo (medicina, historia de la medicina y crítica literaria) también garantizaba la moderación ideológica y el ambiente de relativa tolerancia y libertad vigente en la revista. No obstante, el aspecto más novedoso de la filosofia editorial y el funcionamiento práctico de Cuadernos Hispanoamericanos era la puesta de relieve y la puesta en práctica consecuente del diálogo. Naturalmente, dadas las circunstancias políticas, la gran mayoría de los intelectuales latinoamericanos que se incorporaron a este diálogo pertenecían a corrientes más moderados o conservadores. A pesar de estas limitaciones ideológicas, es importante subrayar que en vez de una plataforma ideológica prefabricada, la revista funcionaba como un laboratorio ideológico y cultural relativamente abierto. El primer director de la revista, Pedro Laín Entralgo, también subrayó la importancia del diálogo en su saludo editorial del primer número de Cuadernos Hispanoamericanos, publicado en 1948:

,Quien lea esta revista debe saber, ante todo, que ba nacido para servir al diálogo. El bombre vive humanamente en cuanto dialoga consigo mismo, con los demás bombres, con la Divinidad. [...] Nosotros, los hacedores y lectores de estos neonatos cuadernos, vamos a servir bispánicamente a ese hondo, esencial imperativo del diálogo. Dos modos diversos asumirá nuestro servicio. Seremos, por una parte, área, bogar de diálogo. Viviremos, por otra, con voluntad, con intención de diálogo. [...] Españoles e hispanoamericanos hemos de contarnos muchas cosas aceria de nuestro modo de ver, sentir, pensar y cantar el mundo y los problemas bumanos, de nuestra bistoria comin y diversa, de nuestros dolores y esperanzas, de nuestras ambiciones. [...] Queremos ser algo más que espacio neutral, lonja inerte donde las voces resuenen con esa mansa y honda resonaniza de la letra escrita. También para nosotros el diálogo es vivir. Dialogaremos amistosamente con todos cuantos quieran ser fieles al modo de ser que llamamos Hispanidad, es decir, a la mejor posibilidad histórica de los hombres españoles $e$

\footnotetext{
${ }^{15}$ Cuadernos Hispanoamericanos (Kraus Reprint), №.19, 1951.

${ }^{16}$ CAPELATO, op. cit., 345.

${ }^{17}$ Javier Tusell, Manual de Historia de España, tom. VT., El siglo XX, Madrid, Historia 16, 1990, 654.
} 
bispanoamericanos. Dialogaremos polémicamente con todos los enemigos y disidentes de ese alto modo de ser hombre y de su derecho a la existenicia histórica." 18

¿Cómo han visto, entonces, los participantes de este diálogo práctico y orgánico (aúnque de límites ideológicos claros), los autores españoles y latinoamericanos de Cuadernos Hispanoamericanos, el balance de la independencia latinoamericana, y cómo opinaron sobre las perspectivas del subcontinente latinoamericano a finales de la década de los cuarenta y a comienzos de la década de los cincuenta? Parece que los intelectuales de ambos continentes tenían interpretaciones parecidas acerca de unos aspectos importantes de los procesos históricos de la América Latina independiente y de España. En un artículo de 1948 de Cuadernos Hispanoamericanos, el historiador y politólogo argentino, César F. Pico subrayó que en el siglo XIX, el mundo hispano, es decir, tanto los países de América Latina como España pasaron a la periferia de la comunidad internacional, $\mathrm{y}$, como consecuencia, las ideas del racionalismo llegaron a tener una influencia considerable en la totalidad de la configuración social y política en los países de estas regiones con un retraso considerable:

'Tanto en ellas [las colonias latinoamericanas - A. V.] como en la metrópoli, el racionalismo penetró tardiamente en nuestros buertos cerrados. Fue un intento, no del todo logrado, de suplantar las antiguas creencias sociales en la nueva fe en la razón." 19

Aparte de los inconvenientes evidentes de esta evolución en una comparación internacional, Pico también identifica algunas ventajas de la situación. Según la opinión del autor, cómo resultado posterior de la relativamente tarde llegada del racionalismo, tanto para las sociedades de América Latina como para la sociedad española era más fácil liberarse de los modos de pensar del racionalismo ya entrado en crisis, que paralizaron, en su opinión, a las demás sociedades de Occidente de la época y resultaron en una infecundidad intelectual relativamente generalizada en la civilización:

'La ventaja consiste en el mencionado retardo, en la franquia con que aguardamos los venturos tiempos, libres de los hábitos mentales impresos por el racionalismo y que puede sobrevivir después del naufragio de este último." 20

Los autores también parecen estar de acuerdo sobre el papel de España después de que cesó de ser una metrópoli. Hay que subrayar que las opiniones reflejadas también suponen cierta actitud autocrítica por parte de los autores españoles. En el mismo artículo anteriormente mencionado, César E. Pico analiza la influencia internacional de España después de la independencia de sus colonias latinoamericanas, y llega a la conclusión de que aparte de las evidentes circunstancias políticas, la fuerte matiz individualista, siempre presente, en su opinión, en la consciencia colectiva española, también tenían mucha influencia en la pérdida de importancia internacional del país. Sin

18 Pedro LAÍN ENTRALGO, "A quién leyere", in: Cuadernos Hispanoamericanos (Kraus Reprint), 1, 1948, 7-8.

${ }^{19}$ César E. PICO, "Nuestro tiempo y la misión de las Españas", in: Cuadernos Hispanoamericanos (Kraus Reprint), 1, 1948, 54.

20 PICO, op. cit., 55. 
embargo, el autor llama la atención al hecho que el mismo individualismo puede salvar al país del peligro de la expansión comunista y de las ambiciones totalitarias:

'Frente a los progresos de la socialización que amenazan al mundo y que acarrean el riesgo de absorber al hombre en la termitera colectiva quitándole incluso la responsabilidad de sus actos y arrancándole su destino personal, el individualismo español se presenta como una de las más benditas esperanzas para todos aquellos que advierten aterrados el pavoroso peligro." 21

En un ensayo sobre la vocación histórica de España, Pedro Laín Entralgo matiza la presencia y el peso relativo del país en la esfera internacional, desde una perspectiva histórica. El autor resalta que España no desapareció del escenario internacional: debido a sus conflictos con otros estados, a lo largo de todo el siglo XIX, no dejó de ser un actor con considerable visibilidad, pero perdió su anterior rol de protagonista, dejó de ser un factor determinante en la evolución de la política internacional:

"España calzó otra vez el coturno trágico, pero el escenario de la tragedia cambió, reducido del planeta entero a la Peninsula Ibérica." 22

Por otra parte, varios autores subrayaron la creciente importancia de América Latina, el otro componente del mundo hispanoamericano, en el sistema internacional. Por ejemplo, el filósofo y político mexicano, José Vasconcelos, razona que a causa de la determinación externa de los procesos históricos, los pueblos de América Latina fueron excluidos de la formación de la historia universal. No obstante, debido a la crisis estructural después de la Segunda Guerra Mundial, están obligados a participar activamente en la tarea de salvar el futuro histórico de la humanidad:

"Silencioso y profundo ha de ser el iberoamericano abora: paciente, porque le tocó pertenecer a pueblos que no baien la bistoria, $y$, sin embargo, deben colaborar para salvarla. Silencioso, porque guarda un secreto que inspira confianza: la promesa implicita en el verso del poeta que advirtio: "mi hora no ha llegado todavia.' [...] La conicencia iberoamericana situada en la región del no tiempo, es en el mundo presente la más indicada para juzgar bombres y cosas. No la ofuscan nacionalismos ni periuicios, puesto que su liga con el pasado es agradecida, pero no parial. Ignora el resentimiento porque sabe que su destino pertenece al futuro, $y$ no le falta uno solo de los haces de la simpatía, porque en su constitución han entrado y siguen entrando las simientes todas de lo bumano: España y Portugal, Europa toda, en parte, y lo aborigen, más una porión considerable de lo africano y poio de lo asiático." 23

El tema del mestizaje también aparece en el ensayo anteriormente citado, y merece atención aparte cómo los autores de Cuadernos Hispanoamericanos interpretaron y evaluaron el proceso de mestizaje, crucial en la formación y en la consolidación de los de los estados latinoamericanos y también un factor importante en su consiguiente evolución social. Otra vez tenemos que subrayar que las posibles concordancias entre los autores españoles y latinoamericanos en este tema son debidas principalmente a la orientación ideológica de los autores latinoamericanos, representantes, en su mayoría, de las corrientes del

${ }^{21}$ Ibidem, 57.

22 Pedro LAÍN ENTRALGO, "Hispanidad y Modernidad", in: Cuadernos Hispanoamericanos (Kraus Reprint), 2, 1948, 294.

${ }^{23}$ José VASCONCELOS, "Deber de Hispanoamérica", in: Cuadernos Hispanoamericanos (Kraus Reprint), No.1, 1948, 108-109. 
conservadurismo o del nacionalismo moderado. Es notable la intensidad con la cual persiste la argumentación contra el legado de la Leyenda Negra en relación con el tema racial - o, en el caso español, sobre las características nacionales tipificadas:

'Representa el mérito y la culpa de este país el baber ireado un nuevo prototipo, el bombre señor. El rasgo sobresaliente de este ideal español reside en el sensible y celoso sentido del honor, en cuyo nombre $y$ a cuyo servicio todo, basta el dar muerte, estaba permitido, exigido y glorificado." - explica Karl Vossler los orígenes de la imagen negativa estereotipada del español ${ }^{24}$.

Los autores de la revista contrapusieron con preferencia la tolerancia racial del mundo hispano con el racismo, en su interpretación todavía extendido, en los Estados Unidos. Asimismo, varios colaboradores, como, por ejemplo, Osvaldo Lira, resaltaron que la propaganda anti-española tendió a utilizar el ejemplo del fenómeno antropológico del mestizaje racial y cultural en América Latina para resaltar la marcada distancia y los varios elementos etnológicos y culturales entre la metrópoli antigua y las repúblicas latinoamericanas independientes. Siendo un defensor de la Hispanidad, Lira intenta refutar esta alegación con un rotundo argumento:

'El mestizaje está muy lejos de poseer, en el orden de la inteligencia y de la cultura, que es el orden especifico de las naciones, la importancia que se le atribuye. En consecuencia, sea lo que fuere de las aportaciones indígenas, así como de las de los europeos establecidos en los pocos territorios de América, las naciones hispanoamericanas fueron en un principio y ban continuado sin interrupión hasta nuestros dias esencialmente españolas." 25

El joven Manuel Fraga Iribarne, otro regular en las columnas de Cuadernos Hispanoamericanos, también dedicó una serie de artículos en la revista al tema del racismo y de la cuestión racial, en los cuales analizó y reseñó en detalle las principales obras contemporáneas dedicadas al tema. El punto de vista de Fraga es interesante, ya que representa una versión moderada de la ideología oficial de la Hispanidad. En contraste con la interpretación anteriormente citada de Osvaldo Lira, la cual alega la influencia española exclusiva en la construcción racial y cultural de los pueblos de hispanoamericanos, Fraga Iribarne subraya las tradiciones y la vocación compartidas por estos pueblos:

"Tal es la América de boy, mestiza, india y española a la vez, en la que cabe una fe racial de la Hispanidad; basado en la consigna de 'ser para bacer... y bacer para ser', porque si desde un punto de vista naturalista las razas son para hacer, naturalmente las razas bacen, 'actuan para llegar a ser.' " 26

Es decir, el autor considera que todos los pueblos del mundo hispanoamericano forman una totalidad orgánica, indivisible según las categorías históricas o antropológicas, ya que su esencia es abstracta, arraigada en un estilo de vida colectivo (tradiciones, valores culturales y morales), la espiritualidad colectiva de la Hispanidad, y

${ }^{2+}$ Karl VOSSLER, "Sobre la mentalidad del español", in: Cuadernos Hispanoamericanos (Kraus Reprint), 21, 1951, 347.

25 Osvaldo LIRA, "Hispanidad y mestizaje", in: Cuadernos Hispanoamericanos (Kraus Reprint), 8, $1948,279-280$.

26 Manuel FRAGA IRIBARNE, "Razas y Racismo", in: Cuadernos Hispanoamericanos (Kraus Reprint), 14, 1950, 237. 
por la misma razón, según sus interpretaciones, el fenómeno del racismo es inexistente tanto en España como en América Latina ${ }^{27 .}$

Aparte del análisis del proceso histórico, cultural y social de los países latinoamericanos después de la Independencia, y de sus relaciones con España, los autores de Cuadernos Hispanoamericanos también seguían con atención los acontecimientos políticos contemporáneos del subcontinente latinoamericano. Como ya hemos indicado, la sección "Nuestro tiempo" de la revista era dedicada al análisis de los acontecimientos políticos importantes de la actualidad, y los temas latinoamericanos, sobre todo, la evolución de la integración regional de los países de América Latina, y las cumbres y reuniones de los organismos de integración estaban bien representadas en esta sección. En el período examinado, los principales acontecimientos políticos del carácter multilateral mencionado eran la IX Conferencia Panamericana, celebrada en 1948 en Bogotá28, y la IV Reunión de Cancilleres americanos en Washington, en 1951. Los colaboradores de la revista contemplaron con optimismo la institucionalización de la integración latinoamericana, a pesar de que hubiera sido realizada bajo el liderazgo de los Estados Unidos. Analizando las tareas de la IX Conferencia Panamericana, Mario Amadeo llama la atención a la singular importancia de la recopilación de la nueva carta constitutiva del sistema interamericano, futuro fondo orgánico legislativo de la cooperación entre los países de América Latina y Estados Unidos ${ }^{29}$. Otro autor anónimo esboza el futuro de la cooperación interamericano a través del ejemplo de la creciente cooperación económica entre Venezuela, Colombia y Ecuador, antiguos miembros de la Gran Colombia (manifestación regional práctica del panamericanismo bolivariano), y afirma que "No está lejano el día en que los "Estados Desunidos de la América del Sur» se agrupen en unidades que aciaben por reducirse a la unidad." 30

Naturalmente, tampoco faltaron las evaluaciones más críticas de los verdaderos propósitos de la cooperación interamericana y de la posición desigual de negociación de los países latinoamericanos frente a los Estados Unidos. En su artículo anteriormente mencionado, Mario Amadeo llama la atención al fenómeno que los intereses de las potencias económicas del hemisferio occidental, de Argentina y de los Estados Unidos, dominan en la cooperación económica interamericana, y la codificación de los acuerdos en este terreno puede resultar en el hecho que la práctica ya existente y difundida de otorgar facilidades a la inversión extranjera (predominantemente estadounidense) se convierta en una obligación jurídica ineludible para los países latinoamericanos ${ }^{31}$.

\footnotetext{
${ }^{27}$ Ibidem, 238.
}

${ }^{28} \mathrm{La}$ conferencia dio lugar a la creación de un nuevo organismo internacional de integración regional, la Organización de los Estados Americanos, el principal foro multilateral del hemisferio occidental. Véase: Juan Carlos PEREIRA (coord.), Diccionario de relaciones internacionales y palitica exterior, Barcelona, Ariel, 2008, 694-695.

${ }^{29}$ Mario AMADEO, "Perspectivas de la Conferencia de Bogotá", in: Cuadernos Hispanoamericanos (Kraus Reprint), 2, 1948, 263.

30 Anónimo, "La 'Carta de Quito' y la conferencia económica grancolombiana", in: Cuadernos Hispanoamericanos (Kraus Reprint), 4, 1948, 75-76.

31 AMADEO, op. cit., 267. 
Asimismo, en su artículo sobre los principales acontecimientos de la IV. Reunión de de Cancilleres Americanos en Washington, A. A. Largo Carballo resalta que desde un punto de vista más realista, las metas y los intereses de Estados Unidos y de los países son distintos y poco compatibles. Desilusionado por el estilo del realpolitik, en auge debido al recrudecemiento de la Guerra Fría, el autor describe las sesiones de la reunión como ".. un constante regateo, sin altura ni grandeza" 3 ?.

Pese a las críticas ocasionales sobre el proceso político de la región latinoamericana, podemos establecer que a mediados del siglo XX, los colaboradores latinoamericanos y españoles de la revista Cuadernos Hispanoamericanos estaban de acuerdo que el futuro pertenecería a América Latina - y esperaron que España, ya no cómo superior, sino como aliado igual, también estuviera presente en este futuro. Vieron el subcontinente como un bloque de poder regional en ascensión, dispuesto a reclamar una mayor autonomía y presencia en el escenario internacional, después de un largo tiempo de marginación. Es notable la frecuencia con la cual los autores utilizan la expresión 'el Redescubrimiento de América Latina', e incluso la metáfora de la nueva Atlántida', representando el subcontinente latinoamericano y sus lazos con España, es recurrente en varios textos; la introducción anteriormente citada del editor de la revista, Pedro Lain Entralgo, también contiene esta referencia:

'Fue América para el Viejo Mundo, por obra de españoles, una Atlántida inesperada, un mundo nuevo, milagrosamente interpuesto entre la dulie costa de Huelva y la costa fabulosa de Catay. Perm los buenos europeos, desde Isabel la. Católica y Nebrija, siguieron, seguimos creyendo en la antigua Atlántida. Pero nuestra Atlántida ha de ser creada, no descubierta. No será completa la redondez histórica del orbe mientras los europeos y los americanos no bayamos sabido crear entre Europa y América, sobre el dorso inquieto de las olas atlánticas, una insula ideal, el ágora donde, por virtud de inteligencia y amor, divinos arquitectos, tenga suelo firme nuestra ineludible solidaridad histórica y humana." 33

Naturalmente, las interpretaciones de los autores latinoamericanos y españoles acerca de la participación de España en este proceso eran más heterogéneas. En el artículo anteriormente mencionado, el chileno César E. Pico razona que siendo España el "guardián del depósito de las certezas divinas y de lo que hay permanente en la moral bumana" 34, el país debe renunciar sus vocaciones más laicas como sus ambiciones de influencia política en la región de América Latina, ya que estos afanes de poder son incompatibles con su función moral. Según Pico, sólo de esta manera se puede salvar los valores custodiados por España para el nuevo orden mundial venidero, más harmonioso y justo ${ }^{35}$.

Manuel Fraga Iribarne ofrece una interpretación más práctica y reverberante de la interpretación clásica de la Hispanidad. Fraga analiza el papel de España en relación con América Latina y en el sistema internacional reservando más protagonismo para el país, sobre todo, en la primera relación bilateral. Su artículo también refleja los proyectos

32 A. A. LARGO CARBALLO, "Notas a la IV Reunión de Cancilleres en Washington", in: Cuadernos Hispanoamericanos (Kraus Reprint), 21, 1951, 122-123.

${ }^{33}$ LAIIN ENTRALGO, op. cit., 9.

${ }^{34}$ PICO, op. cit., 60.

35 Ibidem, 61. 
contemporáneos de la diplomacia española de crear, siguiendo el ejemplo del Commonwealth británico, una Comunidad Hispánica de $\mathrm{Naciones}^{36}$, una confederación transatlántica basada en el ámbito cultural compartido, para contrarrestar la influencia de los Estados Unidos:

'Hispanoamérica no consiente que nadie, ni aun los Estados Unidos de Norteamérica, la consideren objeto (y no sujeto) de la politica internacional, o económica, o cultural. Las fórmulas no importan: Hispanoamérica no puede ser coro o familia pobre, como alguien pudo imaginar a lo que entonces llamaban 'las Repúblicas latinoamericanas'. Esta comunidad hispánica de naciones será, por su nueva constitución, uno de los 'nosotros', una de las grandes 'superpotenizas' de nuestro tiempo, en todos los ámbitos de la vida bistórica." 37

En medio del resurgimiento de las ambiciones españolas acerca del subcontinente latinoamericano, era un intelectual chileno, Armando Roa, quién formuló, probablemente con mayor objetividad, las condiciones de una relación de partes iguales entre España y los páses de América Latina. En las conclusiones de un ensayo dedicado a la comparación y el análisis de la evolución de conciencia histórica colectiva de España y América Latina, Roa afirmó que el subcontinente latinoamericano tiene que encontrar su propia voz, tanto en su historia como en su cultura y su literatura, para poder profundizar los lazos con España y "...encontramos al fin con el resto del mundo ocidental" 38.

36 PECHARROMÁN, op. cit., 307.

${ }^{37}$ Manuel FRAGA IRIB $A R N E$, "La hora de Hispanoamérica", in: Cuadernos Hispanoamericanos (Kraus Reprint), 22, 1951, 87-88.

38 Armando ROA, "España y América", in: Cuadernos Hispanoamericanos (Kraus Reprint), 19, 1951. 66-67. 